\title{
PROBABILISTIC LOAD-DEPENDENT CASCADING FAILURE WITH LIMITED COMPONENT INTERACTIONS
}

\author{
Ian Dobson \\ ECE Department \\ University of Wisconsin \\ Madison WI 53706 \\ dobson@engr.wisc.edu
}

\author{
Benjamin A. Carreras \\ Oak Ridge National Lab \\ Oak Ridge TN 37831 \\ carrerasba@ornl.gov \\ David E. Newman \\ Physics Department \\ University of Alaska \\ Fairbanks AK 99775 \\ ffden@uaf.edu
}

\begin{abstract}
We generalize an analytically solvable probabilistic model of cascading failure in which failing components interact with other components by increasing their load and hence their chance of failure. In the generalized model, instead of a failing component increasing the load of all components, it increases the load of a random sample of the components. The size of the sample describes the extent of component interactions within the system. The generalized model is approximated by a saturating branching process and this leads to a criticality condition for cascading failure propagation that depends on the size of the sample. The criticality condition shows how the extent of component interactions controls the proximity to catastrophic cascading failure. Implications for the complexity of power transmission system design to avoid cascading blackouts are briefly discussed.
\end{abstract}

\section{INTRODUCTION}

Industrialized society depends heavily on complicated infrastructure systems with many interconnected components. These infrastructures can suffer widespread failures when stressed components fail successively, with each failure further stressing the system and making further failures more likely. For example, a long, intricate cascade of events caused the August 2003 blackout of a substantial portion of the electrical power system of Northeastern North America affecting fifty million people. The vital importance of the electrical power infrastructure to society motivates the study of models that capture salient features of cascading failure.

I. Dobson and B.A. Carreras gratefully acknowledge coordination of this work by the Consortium for Electric Reliability Technology Solutions and funding in part by the Assistant Secretary for Energy Efficiency and Renewable Energy, Office of Power Technologies, Transmission Reliability Program of the U.S. Department of Energy under contract 9908935 and Interagency Agreement DE-A1099EE35075 with the National Science Foundation. I. Dobson, D.E. Newman, and B.A. Carreras gratefully acknowledge support in part from NSF grants ECS-0214369 and ECS0216053. Part of this research has been carried out at Oak Ridge National Laboratory, managed by UT-Battelle, LLC, for the U.S. Department of Energy under contract number DE-AC05-00OR22725.
Previous work $[3,4,5]$ introduced a probabilistic model of cascading failure with a large number of identical components called CASCADE. The components fail when their load exceeds a threshold, and become more loaded when any other component fails. The components initially have a random load and the cascade is started by an initial disturbance increasing the loading of all components. The number of components failed is a measure of the size of the cascade and it has an analytic probability distribution (a saturating form of the quasibinomial distribution). The CASCADE model can be approximated by a saturating Poisson branching process [6] and the relation of these models to cascading failure in simulated blackouts of power transmission systems is studied in $[2,3]$. CASCADE is an abstract model of cascading failure and one of its purposes is helping to explain the results of power system models of cascading failure blackouts that represent the transmission network and generation redispatch $[1,3]$.

The CASCADE model (and its branching process approximation) show interesting behavior as the average initial component load is increased. In one scenario, as this loading is increased, the average number of failures sharply increases at a critical loading. Moreover, at this critical loading, the probability distribution of the number of failures has a power tail of exponent approximately -1.5 . The critical loading marks a phase transition and an operational boundary with respect to cascading failure. That is, the risk of cascading failure becomes significant at or above the critical loading. Studying this criticality and finding ways to monitor and detect the corresponding criticality in more detailed simulation models or in real infrastructure systems is a promising new direction of research [6,2].

One significant limitation of the CASCADE model is the assumption that all components interact. That is, when one component fails, the loading of all other components is increased. In applications such as blackouts, many thousands of components can interact by a variety of mechanisms and the interactions can sometimes span the entire system. However, it is more realistic to assume that when one component fails, it interacts with only a subset of the 
other components. This paper generalizes the CASCADE model to this limited interaction case and derives the new criticality condition from the branching process approximation to the generalized model. The result has implications for the interesting question of whether new system technologies that improve system performance by increased communication and coordination between system components introduce many unlikely failure modes that could increase the risk of catastrophic cascading failure [8].

\section{CASCADE MODEL WITH $k$ INTERACTIONS}

This section summarizes the generalized CASCADE model. There are $n$ identical components with random initial loads. For each component the minimum initial load is $L^{\mathrm{min}}$ and the maximum initial load is $L^{\max }$. Component $j$ has initial load $L_{j}$ that is a random variable uniformly distributed in $\left[L^{\min }, L^{\max }\right] . L_{1}, L_{2}, \cdots, L_{n}$ are independent.

Components fail when their load exceeds $L^{\text {fail }}$. When a component fails, a fixed amount of load $P$ is transferred to $k$ samples of the $n$ components. The sampling is uniform so that the probability of choosing a particular component is $1 / n$ and the components are sampled independently and with replacement. Moreover, the $k$ samples are chosen independently for each failure.

To start the cascade, an initial disturbance loads $k$ samples of the components by an additional amount $D$. Other components may then fail depending on their initial loads $L_{j}$ and the failure of each of these components will distribute an additional load $P \geq 0$ that can cause further failures in a cascade.

It is useful to normalize the model so that $L^{\mathrm{min}}$ becomes zero and both $L^{\max }$ and $L^{\text {fail }}$ become one $[4,5]$. The normalized initial load $\ell_{j}=\left(L_{j}-L^{\min }\right) /\left(L^{\max }-L^{\min }\right)$ so that $\ell_{j}$ is a random variable uniformly distributed on $[0,1]$. Let

$$
p=\frac{P}{L^{\max }-L^{\min }}, \quad d=\frac{D+L^{\max }-L^{\text {fail }}}{L^{\max }-L^{\min }}
$$

Then $p$ is the amount of load increase on any component when one other component fails expressed as a fraction of the load range $L^{\max }-L^{\mathrm{min}}$. $d$ is the initial disturbance shifted by $L^{\text {max }}-L^{\text {fail }}$ expressed as a fraction of the load range. (The shift ensures that the failure load is one $[4,5]$.)

The model produces failures in stages $i=0,1,2, \ldots$ where $M_{i}$ is the number of failures in stage $i$. It is convenient to state the normalized version of the algorithm. This can be obtained from [4] by adding the random sampling.

\section{Algorithm for normalized CASCADE with $k$ interactions}

0 . All $n$ components are initially unfailed and have initial loads $\ell_{1}, \ell_{2}, \cdots, \ell_{n}$ determined as independent random variables uniformly distributed in $[0,1]$.

1. Uniformly sample components $k$ times independently with replacement and add the initial disturbance $d$ to the load of a component each time it is sampled. Initialize the stage counter $i$ to zero.

2. Test each unfailed component for failure: For $j=$ $1, \ldots, n$, if component $j$ is unfailed and its load $>1$ then component $j$ fails. Suppose that $M_{i}$ components fail in this step.

3. Independently for each of the $M_{i}$ failures, uniformly sample components $k$ times independently with replacement and add $p$ to the load of a component each time it is sampled.

4. Increment the stage counter $i$ and go to step 2 .

\section{BRANCHING PROCESS APPROXIMATION}

In a Poisson branching process model of cascading failure, failures are produced in stages. Each failure at a given stage produces further next stage failures independently according to a Poisson distribution of rate $\lambda$. This section derives the Poisson branching process approximation of the generalized CASCADE model and shows that $\lambda=k p$. Thus $\lambda=k p$ governs the propagation of failures in the cascading process. The implications are discussed in section 4 . Those readers interested in the details of the approximation in this section should read the simpler case in [6] first.

Consider the end of step 2 of stage $i \geq 1$ in the CASCADE algorithm. The failures that have occurred are $M_{0}=$ $m_{0}, M_{1}=m_{1}, \ldots, M_{i}=m_{i}$, but component loads have not yet been incremented in the following step 3 . Let $T_{j i}$ be the number of times component $j$ is sampled in the $k m_{i}$ samples of step 3 of stage $i$. Then the marginal distributions of $T_{j i}, j=1, \ldots, n$ are binomial so that

$$
\begin{aligned}
& P\left[T_{j i}=t \mid M_{i}=m_{i}\right]=\left(\begin{array}{c}
k m_{i} \\
t
\end{array}\right)\left(\frac{1}{n}\right)^{t}\left(1-\frac{1}{n}\right)^{k m_{i}-t} \text { (2) } \\
& E\left[T_{j i} \mid M_{i}=m_{i}\right]=k m_{i} / n \\
& \operatorname{Var}\left[T_{j i} \mid M_{i}=m_{i}\right]=\left(k m_{i} / n\right)(1-1 / n) \\
& \text { Write } \quad \underline{T}_{n i}=\left(T_{1 i}, T_{2 i}, \ldots, T_{n i}\right), \\
& \underline{M}_{i}=\left(M_{0}, M_{1}, \ldots, M_{i}\right), \quad \underline{T}_{i}=\left(\underline{T}_{n 0}, \underline{T}_{n 1}, \ldots, \underline{T}_{n i}\right) \text {, } \\
& \bar{S}_{i}=M_{0}+M_{1}+\ldots+M_{i}, \quad \Sigma_{j i}=T_{j 1}+T_{j 2}+\ldots+T_{j i}, \\
& \alpha_{j i}=\left\{\begin{array}{c}
0 \quad ; \text { component } j \text { failed before stage } i \\
\frac{p t_{j i}}{1-d t_{j 0}-p \sigma_{j(i-1)}} ; \begin{array}{c}
\text { component } j \text { unfailed at } \\
\text { beginning of stage } i
\end{array}
\end{array}\right. \\
& \phi(x)=\left\{\begin{array}{l}
0 ; x<0 \\
x ; 0 \leq x \leq 1 \\
1 ; x>1
\end{array}\right.
\end{aligned}
$$


Consider unfailed component $j$ and suppose its total stage $i$, step 2 additional load $d t_{j 0}+p \sigma_{j(i-1)}<1$. Then, when conditioned on $\underline{T}_{i-1}=\underline{t}_{i-1}$, the load of component $j$ is uniformly distributed in $\left[d t_{j 0}+p \sigma_{j(i-1)}, 1\right]$. In the following step 3 , the probability that the load increment of $p t_{j i}$ causes component $j$ to fail is $\phi\left(\alpha_{j i}\right)$. Now suppose that $d t_{j 0}+p \sigma_{j(i-1)} \geq 1$. Then the probability that component $j$ fails is $\phi\left(\alpha_{j i}\right)=1$.

When conditioned on $\underline{T}_{i}=\underline{t}_{i}$, the component failures in step 2 of stage $i+1$ are independent and hence $M_{i+1}$ has generating function

$$
E e^{z\left[M_{i+1} \mid \underline{T}_{i}\right]}=\prod_{j=1}^{n}\left(1+(z-1) \phi\left(\alpha_{j i}\right)\right)
$$

Since $P\left[M_{i+1}=m_{i+1} \mid \underline{M}_{i}\right]$

$$
\begin{gathered}
=\sum_{\underline{t}_{i}} P\left[M_{i+1}=m_{i+1} \mid \underline{M}_{i}, \underline{T}_{i}=\underline{t}_{i}\right] P\left[\underline{T}_{i}=\underline{t}_{i} \mid \underline{M}_{i}\right] \\
=\sum_{\underline{t}_{i}} P\left[M_{i+1}=m_{i+1} \mid \underline{T}_{i}=\underline{t}_{i}\right] P\left[\underline{T}_{i}=\underline{t}_{i} \mid \underline{M}_{i}\right] \\
E e^{z\left[M_{i+1} \mid \underline{M}_{i}\right]}=\sum_{\underline{t}_{i}} E e^{z\left[M_{i+1} \mid \underline{T}_{i}\right]} P\left[\underline{T}_{i}=\underline{t}_{i} \mid \underline{M}_{i}\right] \\
=\sum_{\underline{t}_{i-1}} A_{i} P\left[\underline{T}_{i-1}=\underline{t}_{i-1} \mid \underline{M}_{i}\right]
\end{gathered}
$$

where $A_{i}=\prod_{j=1}^{n} \sum_{\underline{t}_{n i}}\left(1+(z-1) \phi\left(\alpha_{j i}\right)\right) P\left[\underline{T}_{n i}=\underline{t}_{n i} \mid \underline{M}_{i}\right]$

$$
=\prod_{j=1}^{n} \sum_{t_{j i}}\left(1+(z-1) \phi\left(\alpha_{j i}\right)\right) P\left[T_{j i}=t_{j i} \mid \underline{M}_{i}\right] .
$$

Define $X_{j i}=d t_{j 0}+p \sigma_{j i}$. Then $X_{j i} \geq 1 \Longleftrightarrow p t_{j i} \geq$ $1-d t_{j 0}-p \sigma_{j(i-1)} \Longleftrightarrow \phi\left(\alpha_{j i}\right)=1$. Using (3) and (4),

$$
\begin{aligned}
E\left[X_{j i} \mid \underline{M}_{i}\right] & =\frac{k d+k p s_{i}}{n} \\
\operatorname{Var}\left[X_{j i} \mid \underline{M}_{i}\right] & =\frac{k d^{2}+k p^{2} s_{i}}{n}\left(1-\frac{1}{n}\right)
\end{aligned}
$$

It is convenient to renumber the components so that components $1,2, \ldots, S_{i}$ are the $S_{i}$ components that have failed in previous stages. Then $\alpha_{j i}=0$ for $j=1,2, \ldots, S_{i}$. Moreover $A_{i}=\prod_{j=S_{i}+1}^{n} B_{j i}$ where

$$
\begin{gathered}
B_{j i}=\sum_{t_{j i}}\left[\left(1+(z-1) \alpha_{j i}\right) P\left[T_{j i}=t_{j i}, X_{j i}<1 \mid \underline{M}_{i}\right]\right. \\
\left.+z P\left[T_{j i}=t_{j i}, X_{j i} \geq 1 \mid \underline{M}_{i}\right]\right] \\
=\left(1+(z-1) \frac{p E\left[T_{j i} \mid X_{j i}<1, \underline{M}_{i}\right]}{1-d t_{j 0}-p \sigma_{j(i-1)}}\right) \\
P\left[X_{j i}<1 \mid \underline{M}_{i}\right]+z P\left[X_{j i} \geq 1 \mid \underline{M}_{i}\right]
\end{gathered}
$$

Let $k p=\lambda$ and $k d=\theta$ and $k / n$ be fixed and let $n, k \rightarrow \infty$ and $p, d \rightarrow 0$. If $E\left[X_{j i}\right]<1$, using (7), (8) and (3),

$$
\begin{aligned}
P\left[X_{j i} \geq\right. & \left.1 \mid \underline{M}_{i}\right] \leq P\left[\left|X_{j i}-E\left[X_{j i}\right]\right| \geq\left|1-E\left[X_{j i}\right]\right| \mid \underline{M}_{i}\right] \\
& \leq \frac{\operatorname{Var}\left[X_{j i} \mid \underline{M}_{i}\right]}{\left(1-E\left[X_{j i} \mid \underline{M}_{i}\right]\right)^{2}} \leq \frac{(n / k)\left(\theta^{2}+\lambda^{2} s_{i}\right)}{\left(n-\theta-\lambda s_{i}\right)^{2}} \rightarrow 0
\end{aligned}
$$

and $E\left[T_{j i} \mid X_{j i}<1, \underline{M}_{i}\right] \rightarrow E\left[T_{j i} \mid \underline{M}_{i}\right]=k m_{i} / n$.

Similarly, if $E\left[X_{j i}\right]>1, P\left[X_{j i}<1 \mid \underline{M}_{i}\right] \rightarrow 0$.

Thus $P\left[X_{j i}<1 \mid \underline{M}_{i}\right] \rightarrow I\left[E\left[X_{j i}\right]<1\right]$ and

$B_{j i} \sim\left(1+\frac{z-1}{n} \lambda m_{i}\right) I\left[E\left[X_{j i}\right]<1\right]+z I\left[E\left[X_{j i}\right]>1\right]$.

Now $E\left[X_{j i}\right]<1 \Longleftrightarrow k d / n+k p s_{i} / n<1 \Longleftrightarrow$ $\theta+\lambda s_{i}<n$.

If $s_{i}<(n-\theta) / \lambda$, since $\left(1+\frac{z-1}{n} \lambda m_{i}\right)^{n-s_{i}} \rightarrow e^{\lambda m_{i}(z-1)}$, $A_{i} \rightarrow e^{\lambda m_{i}(z-1)}$. Moreover, since the limit of $A_{i}$ is independent of $t_{i-1},(6)$ implies that $E e^{z\left[M_{i+1} \mid \underline{M}_{i}\right]} \rightarrow e^{\lambda m_{i}(z-1)}$. If $s_{i}>(n-\theta) / \lambda, A_{i} \rightarrow z^{n-s_{i}}$. Therefore, similarly to [6], we can approximate

$$
\begin{aligned}
& E e^{z\left[M_{i+1} \mid \underline{M}_{i}=\underline{m}_{i}\right]} \approx \\
& \left\{\begin{array}{c}
{\left[e^{\lambda m_{i}(z-1)}\right]^{\dagger}+z^{n-s_{i}}\left(1-\left[e^{m_{i} \lambda(z-1)}\right]^{\dagger}(1)\right) ;} \\
s_{i}<(n-\theta) / \lambda \\
z^{n-s_{i}} ; \quad s_{i}>(n-\theta) / \lambda .
\end{array}\right.
\end{aligned}
$$

where $[p(z)]^{\dagger}$ denotes terms of $p(z)$ of degree $\leq n-s_{i}-1$.

Since $e^{m_{i} \lambda(s-1)}=\left(e^{\lambda(s-1)}\right)^{m_{i}},(10)$ is the distribution of the sum of $m_{i}$ independent Poisson random variables with rate $\lambda$ with saturation occurring when the total number of failures exceeds $n$ [6]. Thus we can consider each failure as independently causing other failures in the next stage according to a saturating Poisson Galton-Watson branching process with rate $\lambda=k p$. (This result is the same for the original CASCADE model, except that in the original CASCADE model, $\lambda=n p[6]$.)

The failures produced by the initial disturbance when $i=0$ can also be approximated by a saturating Poisson distribution with rate $\theta$.

\section{CRITICALITY CONDITION \& IMPLICATIONS}

Galton-Watson branching processes proceed in stages to randomly generate an average of $\lambda$ failures from each failure in the previous stage. It is well known [7] that the criticality condition for branching processes is $\lambda=1$, and this conclusion also applies to saturating branching processes [6] and in particular to the saturating branching process derived in the previous section. $\lambda$ governs the propagation of failures so that for $\lambda<1$ the propagation of failures is likely to be limited, whereas for $\lambda>1$ there is a high probability of propagation of failures to the entire system. Thus criticality 


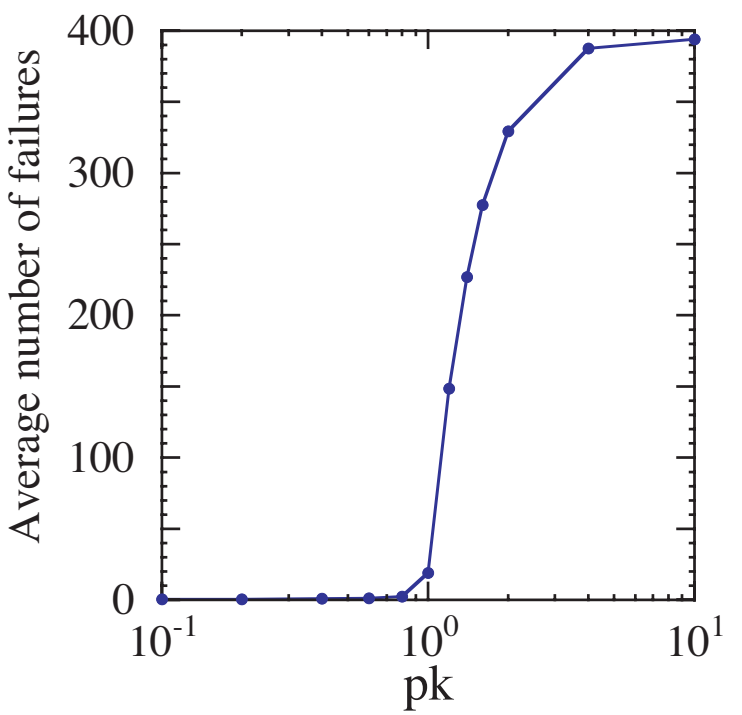

Fig. 1. Average number of failures versus $p k$ as $p$ is varied showing change in gradient at critical point $p k=1$. There are $n=1000$ components and the sample size $k=100$.

in the generalized CASCADE model occurs approximately at

$$
\lambda=k p=1
$$

Simulations of the generalized CASCADE model confirm (11). Figure 1 shows the sharp change at $k p=1$ in the rate of increase of average number of components failed as initial average load is increased. (According to (1), fixing $L^{\max }$ and increasing average initial load $\left(L^{\max }+L^{\min }\right) / 2$ by increasing $L^{\mathrm{min}}$ increases $p$.) Figure 2 shows the power tail at criticality at $k p=1$.

As explained in [6], the risk of cascading failure in these models can be minimized by fixing a design limit $\lambda_{\max }<1$ and requiring $\lambda=k p<\lambda_{\max }$. Then, even if $p$ is very small, large $k$ can cause cascading failure. This suggests that numerous rare interactions between many components can be equally influential in causing cascading failure as a smaller number of likely interactions. Indeed, one can deduce that a design change that introduces a very large number of unlikely failure interactions, thus greatly increasing $k$, could greatly increase the risk of cascading failure, despite the rarity of the failures (low $p$ ). It is conceivable that coupling infrastructures together such as controlling the power transmission system over an internet or certain types of global control schemes could make the system more vulnerable to cascading failure in this fashion. Note that many traditional power system controls are designed to reduce interactions by deliberate separation in distance, frequency, and time scale. Thus the reliability concerns for the effect on cascading failure risk of complicated interconnecting solutions raised by (11) may be consistent with traditional power engineering practice. Our analysis of cascading failure risk is

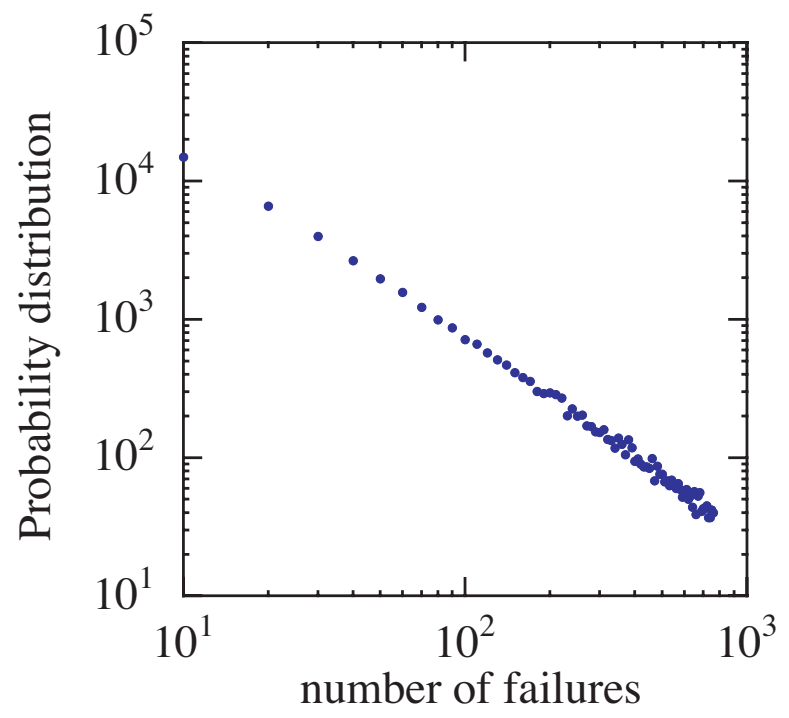

Fig. 2. Probability distribution of number of failures on log$\log$ plot at criticality $k p=1$. There are $n=1000$ components. indeed highly approximate and global in nature, but it starts to quantify trade-offs of complexity versus reliability in engineering large networked systems.

\section{REFERENCES}

[1] B.A. Carreras, V.E. Lynch, I. Dobson, D.E. Newman, Critical points and transitions in an electric power transmission model for cascading failure blackouts, Chaos, vol. 12, no. 4, December 2002, pp. 985-994.

[2] B.A. Carreras, V.E. Lynch, I. Dobson, D.E. Newman, Dynamical and probabilistic approaches to the study of blackout vulnerability of the power transmission grid, 37th Hawaii Intl. Conf. System Sciences, Hawaii, Jan. 2004.

[3] I. Dobson, J. Chen, J.S. Thorp, B.A. Carreras, D.E. Newman, Examining criticality of blackouts in power system models with cascading events, 35th Hawaii Intl. Conf. System Sciences, Hawaii, Jan. 2002

[4] I. Dobson, B.A. Carreras, D.E. Newman, A probabilistic loading-dependent model of cascading failure and possible implications for blackouts, 36th Hawaii Intl. Conf. System Sciences, Hawaii, Jan. 2003.

[5] I. Dobson, B.A. Carreras, D.E.Newman, A loading-dependent model of probabilistic cascading failure, preprint 2003. http:// eceserv0.ece.wisc.edu/ dobson/PAPERS/publications.html

[6] I. Dobson, B.A. Carreras, D.E. Newman, A branching process approximation to cascading load-dependent system failure, 37th Hawaii Intl. Conf. System Sciences, Hawaii, Jan. 2004.

[7] T.E. Harris, Theory of branching processes, Dover NY 1989.

[8] C. Perrow, Normal accidents, updated edition, Princeton University Press, 1999. 